\title{
Factors Suggestive of Cardiac Complications in Takotsubo Cardiomyopathy: Multicenter Study
}

\author{
Tomoko Manno ${ }^{1,2}$, Kenichiro Otomo ${ }^{1}$, Takashi Shibui ${ }^{3}$, Hiroyuki Fujii ${ }^{4}$, Toru Obayashi ${ }^{5}$, Yasuhiro Sato ${ }^{6}$, \\ Osamu Yanase ${ }^{7}$, Mitsuaki Isobe ${ }^{2 *}$
}

${ }^{1}$ Department of Cardiovascular Medicine, Ome Municipal General Hospital

${ }^{2}$ Department of Cardiovascular Medicine, Tokyo Medical and Dental University

${ }^{3}$ Department of Cardiology, Toshima Hospital

${ }^{4}$ Department of Cardiology, Yokohama Minami Kyousai Hospital

${ }^{5}$ Department of Cardiology, Japan Red Cross Musashino Hospital

${ }^{6}$ Department of Cardiology, NHO Disaster Medical Center

${ }^{7}$ Department of Cardiology, Tokyo Metropolitan Otsuka Hospital

*Corresponding author: Mitsuaki Isobe, M.D., PhD, Department of Cardiovascular Medicine, Tokyo Medical and Dental University, Yushima 1-5-45, Bunkyoku, Tokyo-113-8519, Japan, Tel: 81-3-5803-5951; Fax: 81-3-5803-0238;

E-mail: isobemi.cvm@tmd.ac.jp

\begin{abstract}
Background: Takotsubo cardiomyopathy (TM) is characterized by acute and transient myocardial segmental wall motion abnormality especially at the apical area with chest pain mimicking acute myocardial infarction. TM is generally accepted to show good prognosis, however, we experience some cases with severe cardiac complications including acute heart failure (AHF), cardiac rupture and ventricular arrhythmias. We characterized TM patients developing these complications. Methods and results: Eighty three patients were studied. Patients with AHF, cardiac rupture, ventricular arrhythmias and death due to cardiac complications (CC group, $\mathrm{n}=29$ ) were compared with other patients without cardiac complications (NC group, $\mathrm{n}$ $=54)$. There was no difference between the two groups in age, sex, and coronary risk factors. Common primary symptom in the $\mathrm{CC}$ group was dyspnea but was chest pain in $\mathrm{NC}$ group. A preceding emotional stress was a common trigger for CC group. Left ventriculography showed significantly decreased ejection fraction in the CC group (39.7 $\pm 10.2 \%$ vs. $53.9 \pm 14.1 \%, p=0.002$ ). Similarly, more than two segments of impaired left ventricular wall motion was a significant predictor of severe cardiac complications. Conclusions: Clinical onset with dyspnea and extended area of left ventricular ballooning are signs predicting cardiac complications in TM.
\end{abstract}

Received Date: March 29, 2016

Accepted Date: May 02, 2016

Published Date: May 12, 2016

Citation: Isobe, M., et al. Factors Suggestive of Cardiac Complications in Takotsubo Cardiomyopathy: Multicenter Study. (2016) J Heart Cardiol 2(1): 33-38.

DOI: $10.15436 / 2378-6914.16 .023$

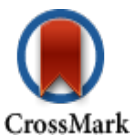

\section{Introduction}

Takotsubo cardiomyopathy (TM) was first described by Sato et al. in $1990^{[1]}$. It is an acute and transient cardiomyopathy characterized by extensive left ventricular systolic dysfunction especially at the cardiac apex and compensatory hyper-contractility of the basal wall. Patients typically present chest pain and electrocardiographic (ECG) changes mimicking acute myocardial infarction ${ }^{[2]}$. The wall motion abnormality usually extends beyond the distribution of a single coronary artery territory, but there are no significant lesions on coronary angiography ${ }^{[3,4]}$. Most patients recover completely within one month. Generally, TM has a good prognosis. The pathophysiological mechanism has long been considered to involve stunning of the myocardium due to multiple coronary artery spasms. However, the hypothesis of myocardial microvascular dysfunction associated with myocardial stunning or spasm is unlikely, considering that such coronary spasms are induced in only about one-third of patients in the chronic stage of TM. Furthermore, histological examination shows the absence of myocardial stunning and the presence of cardiomyopathy and necrosis. Other hypotheses include localized myocarditis, excessive cardiac sympathetic nerve response (disturbance or hyperactivity), neurohumoral factor involvement, coronary artery spasm due to myopericarditis, and myocardial fatty acid metabolism disorders.

Copyrights: (C) 2016 Isobe, M. This is an Open access article distributed under the terms of Creative Commons Attribution 4.0 International License. 
However, the pathogenesis of TM remains to be elucidated. While the prognosis of TM is generally good, some patients develop severe cardiac complications such as cardiac failure, arrhythmia, and cardiac rupture ${ }^{[5-7]}$. TM is a syndrome that has a wide variety of pathologic features, and there is no consensus as to the definition of TM. It varies among researchers. The Mayo Clinic Criteria ${ }^{[8]}$ are the most widely used diagnostic criteria, and have been employed in many studies addressing issues such as clinical characteristics, clinical course, and exacerbating factors in TM patients. In Japan, the Research Committee of Idiopathic Cardiomyopathy funded by the Japanese Ministry of Health, Labour and Welfare proposed diagnostic criteria for TM in $2004^{[9]}$, quite different from those of the Mayo Clinic. The Japanese criteria use a different definition regarding the region of wall motion abnormality and exclude cerebrovascular disorders. To date, only a small number of studies have been conducted in patients with TM based on the Japanese diagnostic criteria. In the present study, we examined clinical characteristics and ECG changes in patients with TM based on the Japanese diagnostic criteria ${ }^{[9]}$ and attempted to identify factors predicting severe cardiac complications.

\section{Methods}

We retrospectively reviewed the clinical characteristics of patients who had been diagnosed with TM at Tokyo Medical and Dental University Hospital and 7 affiliated hospitals during the period between 2001 and November 2010 in order to identify factors associated with severe cardiac complications. The diagnosis was made according to the guidelines for the diagnosis of this form of cardiomyopathy proposed by the aforementioned Research Committee of Idiopathic Cardiomyopathy ${ }^{[9]}$. In principle, patients who had undergone coronary artery angiography were included. Those with ischemic heart disease were excluded. According to the guidelines, TM is defined as a disease exhibiting an acute left ventricular apical ballooning of unknown cause, in which the left ventricle takes on the shape of a jar used for catching octopus, known in Japanese as "Takotsubo".

We also excluded patients with significant organic stenosis or spasm of the coronary arteries, cerebrovascular disorders, pheochromocytoma, and viral or idiopathic myocarditis. The data used for making the diagnosis included clinical symptoms, triggers, age, gender, ECG results, blood test results, and cardiac nuclear images.

We examined the associations between severe cardiac complications or death and clinical factors including patient backgrounds, initial symptoms, ECG results, and other test results. This study was approved by the institutional ethical review board at Tokyo Medical and Dental University, and according to the Ethical Guidelines for Epidemiological Research (Ministry of Education, Culture, Sports, Science and Technology/Ministry of Health, Labour and Welfare), we published all relevant details of this study instead of obtaining informed consent.

Information on symptoms and triggers was obtained by interviewing patients and their families. Baseline clinical characteristics were regarded as present when they were recorded in the hospital charts.

Wall motion analysis with left ventriculography (LVG) was performed using the right anterior oblique projection in which the left ventricle was divided into five segments (\#1 anterobasal, \#2 anterolateral, \#3 apical, \#4 diaphragmatic, and \#5 posterobasal), and the motion of each segment was evaluated in six categories (normal, mild hypokinesis, moderate hypokinesis, severe hypokinesis, akinesis, and dyskinesis) by the physician who performed the test.

Descriptive statistics for clinical characteristics are presented as mean $\pm \mathrm{SD}$ for continuous data and as counts and percentages for categorical data. Univariate analyses were performed using the chi-square test or Student's t test. The variables that were significant on univariate analysis were entered into a multivariate logistic regression model. All analyses were conducted by a physician (Tomoko Manno) with use of JMP ${ }^{\circledR} 12$ software (SAS Institute Inc., Cary, NC). All reported p values are 2-sided. Values are expressed as mean $\pm \mathrm{SD}$.

\section{Results}

In total, 83 patients were included in the analysis. Of these 83 patients, 79 (95.2\%) experienced disease resolution and 4 (4.8\%) died. Of the surviving patients, 25 (30.1\%) experienced severe cardiac complications.

The patients were divided into the cardiac complication group (CC group) and the no-cardiac complication group (NC group). The $\mathrm{CC}$ group consisted of 29 patients who developed severe cardiac complications or died during hospitalization, while the NC group consisted of 54 patients.

Table 1: Cardiac complications

\begin{tabular}{|l|c|}
\hline & $\mathbf{n}(\mathbf{\%})$ \\
\hline Total patients with a complication & $29(34.9)$ \\
\hline Heart failure and cardiogenic shock & $22(26.5)$ \\
\hline Cardiac arrest & $2(2.4)$ \\
\hline Ventricular arrhythmia & $4(4.8)$ \\
\hline Cardiac rupture & $1(1.2)$ \\
\hline
\end{tabular}

As shown in Table 1, the complications in the $\mathrm{CC}$ group were cardiac failure (including cardiogenic shock in 8 ) in 22 patients $(26.5 \%)$, cardiac arrest in $2(2.4 \%)$, ventricular arrhythmia in $4(4.8 \%)$, and cardiac rupture in $1(1.2 \%)$. Of the CC group, 7 patients required mechanical assistance, including percutaneous cardiopulmonary support for 1, intra-aortic balloon pumping for 
5, and electric cardioversion for 1. The patient backgrounds are presented in Table 2. The mean age was $71.9 \pm 11.0$ years. Most patients were women $(n=68,[81.9 \%])$. The initial symptoms were classified into chest pain, dyspnea, consciousness disturbance, and others. Among these, chest pain was most frequent, being experienced by 45 patients (54.2\%). Nine patients (10.8\%) had no symptoms. Triggers were basically classified into physical stress, emotional stress, and uncertain. The physical stress was further classified into infection, post-surgery, hemodialysis, under treatment for another disease, exertion, and alcohol consumption. The proportions of patients with emotional stress and physical stress were nearly equal.

Table 2: Clinical characteristics of all patients

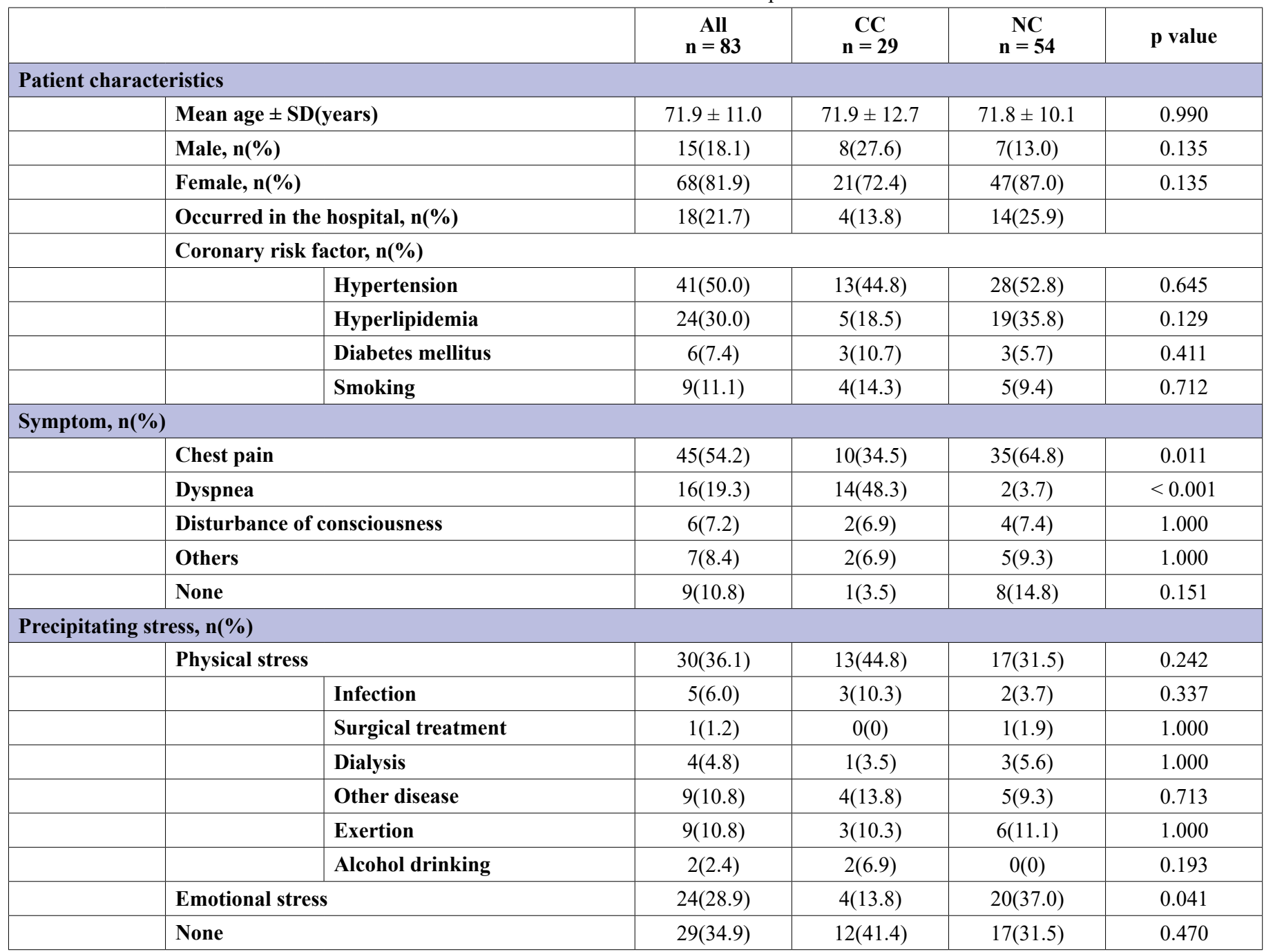

$\mathrm{CC}$ group $=$ patients with cardiac complications, $\mathrm{NC}$ group $=$ patients without cardiac complications

Continuous variables are expressed as mean $\pm \mathrm{SD}$

Comparisons of patient backgrounds between the $\mathrm{CC}$ and $\mathrm{NC}$ groups revealed no significant difference in mean age (71.9 \pm 12.7 vs. $71.8 \pm 10.1$ years; $p=0.99$ ), gender distribution (although there were more women than men in both groups), or coronary risk factors including hypertension, hyperlipidemia, diabetes mellitus, and smoking.

Regarding the initial symptoms, chest pain was more frequently observed in the NC than in the CC group ( $\mathrm{n}=35,[64.8 \%]$ vs. $\mathrm{n}=10,[34.5 \%] ; \mathrm{p}=0.01)$, while dyspnea was more frequently observed in the CC than in the NC group $(\mathrm{n}=14,[48.3 \%] v s . \mathrm{n}$ $=2,[3.7 \%] ; \mathrm{p}<0.001)$.

Table 3: Multivariate analysis of clinical characteristics

\begin{tabular}{|l|c|c|c|}
\hline & Odds ratio & $\mathbf{9 5 \% C I}$ & p value \\
\hline Chest pain & 1.00 & $0.30-3.66$ & 0.998 \\
\hline Dyspnea & 20.4 & $3.90-166.68$ & $<0.001$ \\
\hline Emotional stress & 0.58 & $0.15-1.97$ & 0.394 \\
\hline
\end{tabular}

$\mathrm{CI}=$ confidence interval

Regarding the triggers, emotional stress was significantly more frequent in the NC than in the CC group ( $\mathrm{n}=20,[37.0 \%]$ vs. $\mathrm{n}=4,[13.8 \%] ; \mathrm{p}=0.04)$. Multivariate analyses were performed for the variables that were significant in univariate analysis. 
As shown in Table 3, dyspnea as the initial symptom was an independent predictor of cardiac complications (odds ratio, 20.4; 95\% confidence interval, 3.90-166.68; $\mathrm{p}<0.001$ ).

The results of blood tests and LVG are shown in Table 4. The CC group had significantly higher white blood cell (WBC) count $(12330 \pm 802 / \mu \mathrm{l} v s .9606 \pm 642 / \mu \mathrm{l} ; \mathrm{p}=0.02)$, C-reactive protein $(\mathrm{CRP})$ value $(14.4 \pm 6.6 \mathrm{mg} / \mathrm{dl} v s .3 .5 \pm 1.2 \mathrm{mg} / \mathrm{dl} ; \mathrm{p}=0.05)$, and creatine kinase $(\mathrm{CK})$ value $(1276.8 \pm 321.8 \mathrm{U} / 1$ vs. $712.4 \pm 114.1 \mathrm{U} / \mathrm{l} ; \mathrm{p}=0.03)$ than the NC group.

Table 4: Statistical analysis of laboratory data and left ventriculography

\begin{tabular}{|c|c|c|c|}
\hline & $\mathbf{C C}$ & NC & p value \\
\hline \multicolumn{4}{|l|}{ Laboratory data } \\
\hline $\mathrm{WBC}(/ \mu \mathrm{l})$ & $12330 \pm 802$ & $9606 \pm 642$ & 0.016 \\
\hline CRP(mg/dl) & $14.4 \pm 6.6$ & $3.5 \pm 1.2$ & 0.050 \\
\hline BNP(pg/ml) & $575.2 \pm 88.1$ & $825.1 \pm 247.7$ & 0.958 \\
\hline CK(U/l) & $1276.8 \pm 321.8$ & $712.4 \pm 114.1$ & 0.026 \\
\hline CK-MB(U/l) & $241.3 \pm 30.3$ & $37.4 \pm 23.9$ & 0.075 \\
\hline \multicolumn{4}{|l|}{ Left ventriculography } \\
\hline Ejection fraction(\%) & $39.7 \pm 10.2$ & $53.9 \pm 14.1$ & 0.002 \\
\hline \multicolumn{4}{|l|}{ Wall motion abnormality, $\mathbf{n}(\%)$} \\
\hline$>$ two segment $*$ & $19(82.6)$ & $21(48.8)$ & 0.016 \\
\hline
\end{tabular}

$\mathrm{CC}$ group $=$ patients with cardiac complications, $\mathrm{NC}$ group $=$ patients without cardiac complications

$\mathrm{WBC}=$ white blood cell, $\mathrm{CRP}=\mathrm{C}$-reactive protein, $\mathrm{BNP}=$ brain natriuretic peptide, $\mathrm{CK}=$ creatine kinase, $\mathrm{CK}-\mathrm{MB}=$ creatine kinase $\mathrm{MB}$

Continuous variables are expressed as mean $\pm \mathrm{SD}$

*Cases showing severe hypokinesis, akinesis, or dyskinesis of the left ventricle in at least two segments on left ventriculography.

LVG was performed in 66 patients ( 23 in the $\mathrm{CC}$ and 43 in the $\mathrm{NC}$ group). The mean ejection fraction in the CC group was significantly lower than that in the NC group $(39.7 \pm 10.2 \% v s .53 .9 \pm 14.1 \% ; \mathrm{p}=0.002)$. The proportions of patients who showed decreased wall motion (severe hypokinesis, akinesis, or dyskinesis) in at least two segments was significantly greater in the $\mathrm{CC}$ than in the NC group $(\mathrm{n}=19,[82.6 \%] v s . \mathrm{n}=21,[48.8 \%] ; \mathrm{p}=0.02)$.

ECG was analyzed in 49 patients who had undergone ECG monitoring for one week after the onset (18 for the CC group, and 31 for the NC group). Comparison of ECG between the two groups showed no significant difference in the deepest T-wave inversion $(-1.31 \pm 0.77 \mathrm{mV} v s .-0.97 \pm 0.51 \mathrm{mV} ; \mathrm{p}=0.377)$ or the maximum QTc interval $(0.61 \pm 0.10 \mathrm{msec} v s .0 .56 \pm 0.06 \mathrm{msec}$; $\mathrm{p}=0.07$ ), although the QTc interval tended to be longer in the CC than in the NC group. (Figure 1)
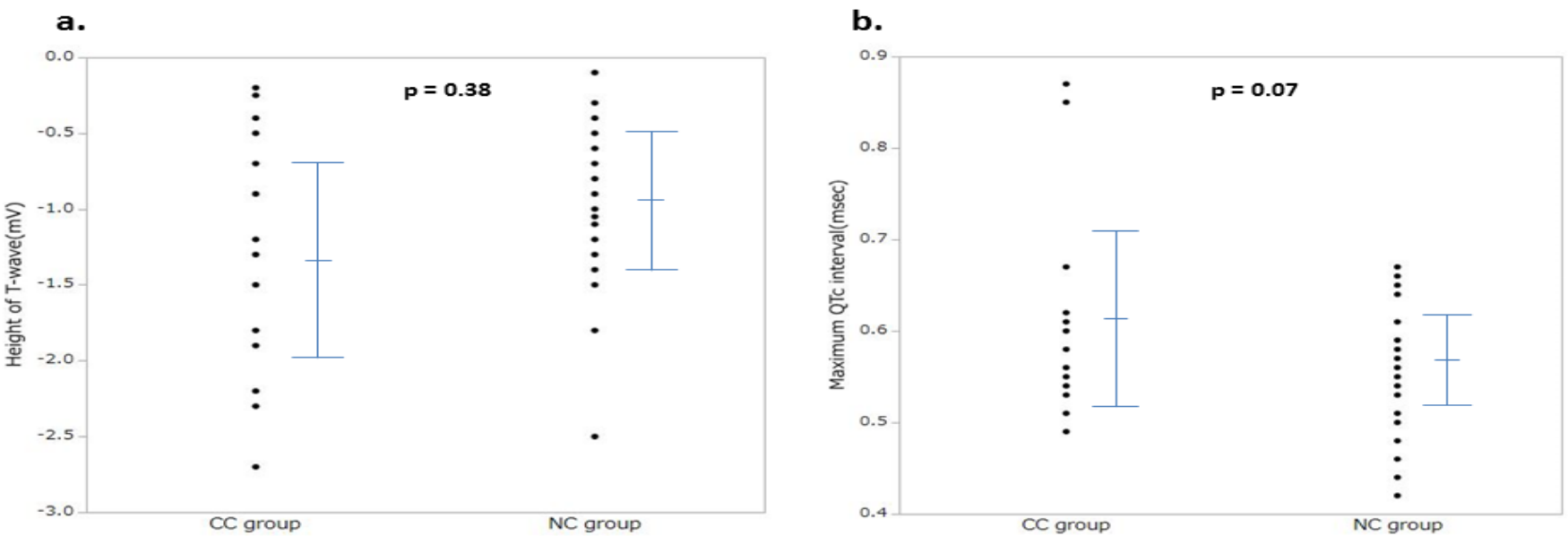

Figure 1: Comparison of electrocardiogram between the two groups

Comparison of electrocardiograms recorded for one week after the onset.

$\mathrm{CC}$ group $=$ patients with cardiac complications, $\mathrm{NC}$ group $=$ patients without cardiac complications

a. The height of the deepest $\mathrm{T}$-wave inversion during the first week were plotted for each group $(-1.31 \pm 0.77 \mathrm{mV} v s .-0.97 \pm 0.51 \mathrm{mV} ; \mathrm{p}=0.377)$.

b. The maximum QTc interval during the first week was plotted for each group $(0.61 \pm 0.10 \mathrm{msec} v s .0 .56 \pm 0.06 \mathrm{msec} ; \mathrm{p}=0.07)$. 


\section{Discussion}

Many recent studies on TM have used the Mayo Clinic Criteria proposed in 2004 (modified in 2008) to make the diagnosis. The Mayo Clinic Criteria are: (1)Transient hypokinesis, akinesis, or dyskinesis of the left ventricular mid-segments with or without apical involvement; the regional wall motion abnormalities extend beyond a single epicardial vascular distribution; a stressful trigger is often, but not always, present; (2) Absence of obstructive coronary disease or angiographic evidence of acute plaque rupture; (3)New ECG abnormalities (ST-segment elevation and/or T-wave inversion) and/or modest elevation of cardiac troponin; (4) Absence of pheochromocytoma or myocarditis ${ }^{[8]}$. In the present study, we used the guidelines for TM diagnosis proposed by the Research Committee of Idiopathic Cardiomyopathy funded by the Japanese Ministry of Health, Labour and Welfare in 2004 ${ }^{[9]}$. The major differences between these two sets of diagnostic criteria are as follows. In the Mayo Clinic Criteria ${ }^{[8]}$, wall motion abnormality is essential but apical involvement is not a requirement. On the other hand, in the Japanese criteria ${ }^{[9]}$, an apical balloon-like dilation is an essential component of TM while cerebrovascular disorders are excluded.

Templin, C. et al. reported that TM can be classified into four different types according to the region affected by wall motion abnormality: apical type, mid-ventricular type, basal type, and focal type ${ }^{[10]}$. It is also known that abnormal ventricular contraction patterns can occur in a wide variety of regions in cerebrovascular disorders ${ }^{[11]}$. In the present study, we excluded patients with cerebrovascular disorders and examined those with apical type, which is the most typical form of TM. We believe that this patient selection might have allowed us to gain a better understanding of the pathology of TM.

It is true that vasospasm of coronary arteries could be associated with the pathogenesis of $\mathrm{TM}^{[3,7,17,18,20,21]}$, but details are still obscure. In the Japanese criteria ${ }^{[9]}$, patients with cardiac vasospasm should be excluded at the discretion of clinicians, but provocation test was not essential examination. In our study, 7 patients (8.4\%) received provocation test of cardiac vasospasm and all of them showed negative results. Tsuchihashi, K. et al. suggested that cardiac vasospasm of TM patients is not necessarily excluded, because assessment of microvascular function is not easy in each patient ${ }^{[3]}$. Therefore, we did not investigate the presence of cardiac vasospasm in all patients thoroughly in this investigation. Further clinical studies are necessary to elucidate the influence of vasospasm on the clinical severity of TM.

Female patients accounted for approximately $80 \%$ of total cases in this study, an observation consistent with those of previous reports. The rates of cardiac complications did not differ significantly between the genders in this study. However, further investigation on this topic may be needed because Murakami and colleagues reported male gender to be an independent predictor of in-hospital composite cardiac events ${ }^{[12-14]}$.

As to the initial symptoms, chest pain was most common, as in previous studies. Among those with severe cardiac complications, dyspnea was most frequent. The results regarding the triggers of TM were consistent with those of many studies showing that physical stress and emotional stress each accounted for $30-40 \% \%^{[15-18]}$, although there is a report indicating that physical stress accounts for $80 \%$ of all cases ${ }^{[19]}$. The present study revealed severe cardiac complications to be less frequent in univariate analysis in patients who experienced emotional stress at the onset.

Currently, blood test data are insufficient, but there are studies showing elevated WBC to be an independent predictor of cardiac events, and a brain natriuretic peptide level $\geq 238 \mathrm{pg} / \mathrm{ml}$ to be an independent predictor of delayed recovery ${ }^{[12,20,21]}$. We found herein that the patients with severe cardiac complications had elevated WBC, CRP, and CK values, suggesting that their myocardial injuries were quite extensive.

LVG showed an association between low ejection fraction and serious complications in this study, an observation consistent with those of previous studies ${ }^{[12,14,15]}$. This study also showed that the risk of serious cardiac complications increased as the region of wall motion abnormality became more extensive. With regard to ECG findings, there is a study showing greater mortality when the QTc interval was $400-550 \mathrm{msec}^{[22]}$. The present results indicate that patients with severe cardiac complications had a prolonged QTc interval, although the difference did not reach statistical significance.

\section{Limitations}

The present study has several limitations. There were many patients who did not undergo coronary angiography in the acute stage of the disease due to background factors such as advanced age. The number of subjects was limited because patients with concomitant ischemic heart disease confirmed by coronary angiography were excluded. This was a retrospective multicenter study, such that blood test results, ECG data, ECG monitoring, magnetic resonance imaging, radioisotope imaging, and ventricular pressure gradient measurement (for those undergoing coronary angiography) were not available in all patients.

\section{Conclusion}

We studied factors predicting severe cardiac complications in patients who had been diagnosed as TM using the diagnostic criteria proposed by the Research Committee of Idiopathic Cardiomyopathy funded by the Japanese Ministry of Health, Labour and Welfare. Comparison of clinical characteristics between those with and without severe cardiac complications (including death) showed association with dyspnea as the initial symptom, an extensive wall motion abnormality region, inflammatory reaction, and elevated cardiac enzymes. Clinicians should be aware that patients with these clinical characteristics have a high risk of developing severe cardiac complications and manage these patients meticulously. 


\section{References}

1. Sato, H., Uchida, T., Dote, K., et al. Tako-tsubo-like left ventricular dysfunction due to multi vessel coronary spasm. In: Kodama, K., Haze, K., Hori, M., eds. Clinical aspect of myocardial injury: from ischemia to heart failure. (1990) Tokyo: Kagakuhyoronsha Publishing: 56-64.22 2. Duran-Cambra, A., Sutil-Vega, M., Fiol, M., et al. Systematic review of the electrocardiographic changes in the takotsubo syndrome. (2015) Ann Noninvasive Electrocardiol 20(1): 1-6.

3. Tsuchihashi, K., Ueshima, K., Uchida, T., et al. Transient left ventricular apical ballooning without coronary artery stenosis: a novel heart syndrome mimicking acute myocardial infarction. (2001) J Am Coll Cardiol 38(1): 11-18.

4. Hurst, R.T., Prasad, A., Askew III, J.W., et al. Takotsubo cardiomyopathy: a unique cardiomyopathy with variable ventricular morphology. (2010) JACC Cardiovasc Imaging 3(6): 641-649.

5. Kurisu, S., Kihara, Y. Tako-tsubo cardiomyopathy: clinical presentation and underlying mechanism. (2012) J Cardiol 60(6): 429-437.

6. Blessing, E., Steen, H., Rosenberg, M., et al. Recurrence of takotsubo cardiomyopathy with variant forms of left ventricular dysfunction. (2007) J Am Soc Echocardiogr 20(4):439.e11-12.

7. Indorato, F., Akashi, Y., Rossitto, C., et al. Takotsubo cardiomyopathy associated with rupture of the left ventricular apex: assessment of histopathological features of a fatal case and literature review. (2015) Forensic Sci Med Pathol 11(4): 577-583.

8. Prasad, A., Lerman, A., Rihal, C. Apical ballooning syndrome (tako-tsubo or stress cardiomyopathy): a mimic of acute myocardial infarction. (2008) Am Heart J 155(3): 408-17.

9. Kawai, S., Kitabatake, A., Tomoike, H. Guidelines for diagnosis of takotsubo (ampulla) cardiomyopathy. (2007) Circ J 71(6): 990-992.

10. Templin, C., Ghadri, J.R., Diekmann, J., et al. Clinical features and outcomes of takotsubo (stress) cardiomyopathy. (2015) N Engl J Med 373(10): 929-938.

11. Masuda, T., Sato, K., Izumi, T. Subarachnoid hemorrhage and myocardial damage from clinical study to animal experiment. (1998) Shinzo 30(1): 21-34.
12. Murakami, T., Yoshikawa, T., Maekawa, Y., et al. Gender differences in patients with takotsubo cardiomyopathy: multi-center registry from Tokyo CCU Network. (2015) PLoS One 10(8): e0136655.

13. Donohue, A., Movahed, M. Clinical characteristics, demographics and prognosis of transient left ventricular apical ballooning syndrome. (2005) Heart Fail Rev 10(4): 311-316.

14. Sharkey, S.W., Pink, V.R., Lesser, J.R., et al. Clinical profile of patients with high-risk tako-tsubo cardiomyopathy. (2015) Am J Cardiol 116(5): 765-772.

15. Pelliccia, F., Parodi, G., Greco, C., et al. Comorbidities frequency in takotsubo syndrome: an international collaborative systematic review including 1109 patients. (2015) Am J Med 128(6): 654.e11-19.

16. Regnante, R.A., Zuzek, R.W., Weinsierb, S.B., et al. Clinical characteristics and four-year outcomes of patients in the Rhode Island takotsubo cardiomyopathy registry. (2009) Am J Cardiol 103(7): 1015-1019. 17. Gianni, M., Dentali, F., Grandi, A.M., et al. Apical ballooning syndrome or takotsubo cardiomyopathy: a systematic review. (2006) Eur Heart J 27(13): 1523-1529.

18. Wang, Y., Xia, L., Shen, X., et al. A new insight into sudden cardiac death in young people: a systematic review of cases of takotsubo cardiomyopathy. (2015) Medicine 94(32): e1174

19. Sharkey, S.W., Windenburg, D.C., Lesser, J.R., et al. Natural history and expansive clinical profile of stress (tako-tsubo) cardiomyopathy. (2010) J Am Coll Cardiol 55(4): 333-341.

20. Shiomura, R., Nakamura, S., Takano, H., et al. Impact of brain natriuretic peptide, calcium channel blockers, and body mass index on recovery time from left ventricular systolic dysfunction in patients with takotsubo cardiomyopathy. (2015) Am J Cardiol 116(4): 515-519.

21. Akashi, Y.J., Goldstein, D.S., Barbaro, G., et al. Takotsubo cardiomyopathy: a new form of acute, reversible heart failure. (2008) Circulation 118(25): 2754-2762.

22. Gopalakrishnan, M., Hassan, A., Villines, D., et al. Predictors of short- and long-term outcomes of takotsubo cardiomyopathy. (2015) Am J Cardiol 116(10): 1586-1590.
Journal ISSN: 2378-6914 (online)

Journal Title: Journal of Heart and Cardiology Short title : J Heart Cardiol
Ommega Online Publishers

E-mail: cardiology@ommegaonline.com

Website: www.ommegaonline.org 